\title{
Tschernobyl - Fukushima: ärztliche Verantwortung in der Atompolitik
}

\section{Martin Walter, \\ Günter Baitsch, \\ Jacques Moser}

Für den Vorstand Physicians for Social Responsibility (PSR) und International Physicians for the Prevention of Nuclear War (IPPNW) Schweiz
«Soll die Medicin daher ihre grosse Aufgabe wirklich erfüllen, so muss sie in das grosse politische Leben eingreifen; sie muss die Hemmnisse angeben, welche der normalen Erfüllung der Lebensvorgänge im Wege stehen, und ihre Beseitigung erwirken.»

(Rudolf Virchow [1])

Ionisierende Strahlung erzeugt maligne Tumoren und genetische Schäden. Das ist bekannt. Weniger bekannt ist, dass Strahlenbelastungen auch transgenetisch schädlich sein können. Kinder von Eltern, die vor der Zeugung bestrahlt wurden, können Tumoren und Genschäden aufweisen [2, 3]. Dafür sprechen viele Indizien auch molekular-biologischer Natur [4]. Teratogenese, Totgeburten und Genominstabilität in den Folgegenerationen sind auf diesem Gebiet weitere Themen [5]. Im folgenden Beitrag wollen wir auf einige spezifische, für Ärzte besonders wichtige Aspekte hinweisen. Es ist Zeit, nach der Havarie von Fukushima die Debatte über ionisierende Strahlung in der Ärzteschaft wieder aufzunehmen.

\section{Die Freisetzung von Reaktormaterial in die Biosphäre}

Ein Reaktorunfall setzt im Falle einer Kernschmelze in der Regel einen mehr oder weniger grossen Teil des radioaktiven Reaktorinventars frei. So ist es geschehen in Tschernobyl und erneut in Fukushima Daiichi in Japan. Dort hat zwar beim Erdbeben die Reaktornotabschaltung funktioniert, die Reaktoren sind aber wegen der Erderschütterung alle in den Inselbetrieb übergegangen, das heisst, sie haben den Anschluss
Reaktorkern muss wegen dieses Zerfallsprozesses, der zur Produktion der Nachzerfallswärme führt, über sehr lange Zeit gekühlt werden. Dadurch soll verhindert werden, dass er zu glühen beginnt, ja sogar schmilzt, was zu einer erneuten, unkontrollierbaren Kettenreaktion und einer exzessiven Energieexkursion führen könnte. Der schmelzende Kern kann durch die Stahlhülle des Reaktorbehälters dringen, oder durch den Betonboden des Containments und mit Grundwasser in Berührung kommen, was zu einer Dampfexplosion führen kann und zur Freisetzung des ganzen Reaktorinventars. Dies entspricht einem Super-GAU.

Bei einer Zerstörung des Sicherheitsbehälters oder bei einer Leckage im Dampf- und Wasserkreislauf nach Kernschmelze (wie in Fukushima in mehreren Reaktoren) werden zuerst gasförmige Stoffe, zum Beispiel Edelgase wie Krypton und Xenon, freigesetzt, ebenfalls leicht flüchtige Stoffe wie Iodisotope, ${ }^{137}$ Cäsium und ${ }^{134}$ Cäsium. Weniger flüchtige Stoffe, ${ }^{90}$ Strontium, Uranisotope und Transurane wie ${ }^{239} \mathrm{Plu}-$ tonium liegen als Partikel (Aerosole) vor, oder sind an Staubteilchen gebunden. Ob von diesen Stoffen das gesamte im Reaktor enthaltene Inventar oder nur Teile freigesetzt werden und wie weit diese transportiert werden, hängt vom Verlauf der Kernschmelze und von den meteorologischen Bedingungen ab. In Fukushima wurde Plutonium in der Umgebung der Reaktoren gemessen. Anzunehmen ist deshalb, dass auch ${ }^{90}$ Strontium, Transurane und weitere Isotope freigesetzt wurden.

\section{«Es gibt keine Toleranz des Organismus für ionisierende Strahlung. Jede Strahlung kann einen Krebs auslösen oder eine Schädigung des Genoms bewirken.»}

ans Stromnetz verloren. Die Notkühlsysteme sind zum Teil angesprungen, Batterien und Dieselmotoren haben über angeschlossene Generatoren Strom für die Kühlpumpen geliefert; die Motoren wurden dann aber durch den Tsunami abgewürgt.

In abgeschalteten Reaktoren fällt die Energieproduktion nicht auf null, obschon die Kettenreaktion gestoppt ist. Die zerfallenden Spaltprodukte in den Brennstäben produzieren weiterhin Wärme. Der
Jedes Isotop hat seine eigene Pharmakokinetik. Bei Mensch und Wirbeltieren verhalten sich ${ }^{137} \mathrm{Cä-}$ sium und ${ }^{134}$ Cäsium wie Kalium. Sie gelangen in alle Zellen. Die Muskelmasse ist beim Menschen besonders gross, weshalb Cäsium zum grossen Teil dort aufgenommen wird, so auch in der Herzmuskulatur. Bandashewski beschrieb eine Kardiomyopathie [6] bei Kindern in Weissrussland in der Folge des Reaktorunfalls von Tschernobyl. 
${ }^{90}$ Strontium verhält sich im Stoffwechsel wie Calcium. Es ist sehr «knochenavid». Als Betastrahler ist ${ }^{90}$ Strontium vor allem für Kinder bedrohlich. Diese Teilchenstrahlung penetriert nicht sehr tief in die Gewebe. Weil aber das kindliche Knochenmark fettarm ist, und das ${ }^{90}$ Strontium sich auch subendostal anlagert, liegt es nahe am blutbildenden Knochenmark und belastet die hämatologischen Stammzellen viel höher als beim Erwachsenen.

${ }^{239}$ Plutonium hat wohl die höchste Radiotoxizität aller Transurane. Die Verweildauer von ${ }^{239}$ Plutonium im menschlichen Organismus ist äusserst lang: einmal im Körper, wird es kaum mehr ausgeschieden. Plutonium penetriert in den Organismus durch Inhalation. Mit der Nahrung eingenommen, passiert ein grosser Teil den Darm, ohne resorbiert zu werden.

In Tschernobyl wurden viele Isotope, auch Transurane freigesetzt, darunter ${ }^{133} \mathrm{Xe},{ }^{131} \mathrm{I},{ }^{134} \mathrm{Cs},{ }^{137} \mathrm{Cs}$, ${ }^{132} \mathrm{Te},{ }^{89} \mathrm{Sr}$, ${ }^{90} \mathrm{Sr},{ }^{140} \mathrm{Ba},{ }^{95} \mathrm{Zr}$, ${ }^{99} \mathrm{Mo},{ }^{103} \mathrm{Ru},{ }^{106} \mathrm{Ru},{ }^{141} \mathrm{Ce}$, ${ }^{144} \mathrm{Ce},{ }^{239} \mathrm{~Np},{ }^{238} \mathrm{Pu},{ }^{239} \mathrm{Pu},{ }^{240} \mathrm{Pu},{ }^{241} \mathrm{Pu},{ }^{242} \mathrm{Cm}$ [7], jedes mit seiner eigenen Pharmakokinetik und spezifischen Radiotoxizität. Ähnliches ist in Fukushima zu erwarten.

Allen aus Reaktoren freigesetzten Isotopen - wie auch den natürlich vorkommenden Strahlenquellen ist gemeinsam die Potenz zur Erzeugung von Krebs, Gefässkreislaufkrankheiten, Endokrinopathien und Schädigung des Erbgutes. Strahlenbiologisch ist gesichert, dass die Dosiswirkungsrelation linear ist. Mit anderen Worten: Es gibt keine Toleranz des Organismus für ionisierende Strahlung. Jede Strahlung kann einen Krebs auslösen oder eine Schädigung des Genoms bewirken.
Fukushima ist nicht Tschernobyl - oder doch? Reflexartig berichteten die Medien, der Unfall in Fukushima sei nicht vergleichbar mit dem in Tschernobyl. Doch stellt sich die Frage: Welcher der beiden Unfälle ist gravierender für die Umgebung? Für die lokale Bevölkerung war der Tschernobylunfall wegen seines Mechanismus (Explosion, Graphitbrand, Kaminbildung, Verteilung der Isotopen über die ganze Nordhalbkugel) im Ganzen gesehen ein Glück im Unglück: Sie bekam nicht alles ab. Die havarierten Fukushimareaktoren hingegen setzen wie ein Schwelbrand alles in der näheren Umgebung ab. Wenn also geschrieben wird, es sei in Fukushima nur etwa 1/10 der in Tschernobyl entwichenen Radioaktivität freigesetzt worden - was pure Spekulation ist, denn niemand weiss es - bedeutet das keineswegs, dass der Unfall für die japanischen Anwohner nicht viel gravierender ist als der von Tschernobyl für die dortige Bevölkerung. Erst nach vielen Jahren wird das wahre Ausmass der Katastrophe bilanzierbar sein.

Nein, Fukushima ist nicht Tschernobyl. In der Sowjetunion haben 600000 Soldaten, Zivilschützer und Reservisten aufgeräumt. In Japan spricht man bisher von 500 Arbeitern, die bis zur physischen und psychischen Erschöpfung zur Arbeit getrieben werden. Sie werden hohen Strahlendosen ausgesetzt. Die Rekrutierung neuer Arbeiter sei schwierig, hören wir heute schon. Wenn die 500 Mann an der Grenze der möglichen Strahlenbelastung angelangt sind (250 mSievert akkumulierte Dosis), wer wird dann die Arbeit weiterführen?

Die Folgen von Tschernobyl sind hinlänglich bekannt, wenn auch nicht genügend untersucht und

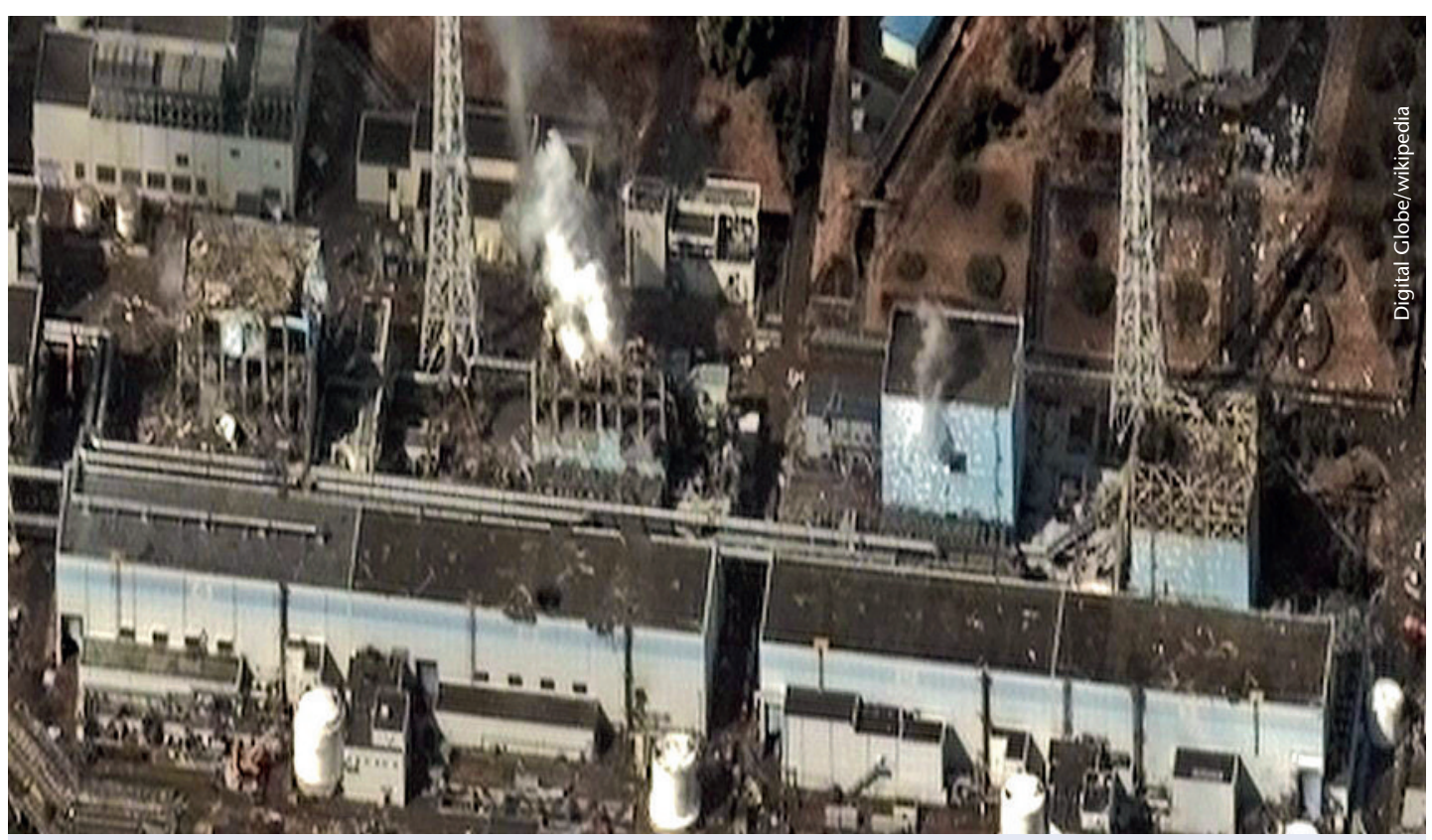

In Fukushima (Aufnahme vom 16.3.2011) wurde Plutonium in der Umgebung der Reaktoren gemessen. Anzunehmen ist deshalb, dass auch ${ }^{90}$ Strontium, Transurane und weitere Isotope freigesetzt wurden. 


\section{Atomunfälle}

Kanada, Dezember 1952: In einem Reaktor in Chalk River bei Ottawa kommt es zu einer schweren Explosion. Der Reaktorkern wird bei einer partiellen Kernschmelze zerstört.

Russland, September 1957: In einer Wiederaufbereitungsanlage in Kyschtym explodiert ein Tank mit radioaktiven Abfällen. Dabei werden grosse Mengen an radioaktiven Substanzen freigesetzt.

Grossbritannien, Oktober 1957: Im Kernreaktor in Windscale - ab 1983 Sellafield genannt - wird nach einem Brand eine radioaktive Wolke freigesetzt, die sich über Europa verteilt.

Schweiz, Januar 1969: Beim Versagen des Kühlsystems eines experimentellen Reaktors im Versuchsatomkraftwerk Lucens kam es zu einer partiellen Kernschmelze. Grossbritannien, Juli 1973: Wieder kommt es in der Wiederaufbereitungsanlage Windscale zu einer schweren Explosion, bei der ein grosser Teil der Anlage kontaminiert wird.

Deutschland, Januar 1977: Kurzschlüsse in zwei Hochspannungsleitungen führen im Atomkraftwerk Gundremmingen in Bayern zu einem Totalschaden. Das Reaktorgebäude ist mit radioaktivem Kühlwasser verseucht.

USA, März 1979: Maschinen- und Bedienungsfehler führen im Kernkraftwerk Three Mile Island bei Harrisburg zum Ausfall der Reaktorkühlung, die eine partielle Kernschmelze und die Freisetzung von radioaktiven Gasen zur Folge hat.

Sowjetunion, April 1986: Explosion und Kernschmelze im Reaktor Nr. 4 des Atomkraftwerkes von Tschernobyl. Der radioaktive Niederschlag kontaminiert grosse Teile der Welt. Das Ausmass der Folgen ist bis heute unklar. Nach einer kürzlichen Publikation der Akademie für Wissenschaften, New York, erreicht jedoch die Zahl der Todesopfer beinahe eine Million.

Japan, September 1999: In einem Brennelementewerk in der Stadt Tokaimura setzt nach einer unvorschriftsmässigen Befüllung eines Vorbereitungstanks eine unkontrollierte Kettenreaktion ein. Starke radioaktive Strahlung tritt aus.

Tschechien, Oktober 2000: Das umstrittene Atomkraftwerk Temelin geht ans Netz. Bis August 2006 werden von der Anlage fast hundert Störfälle gemeldet.

Deutschland, Dezember 2001: Eine Wasserstoffexplosion verursacht im Atomkraftwerk Brunsbüttel einen Störfall. Der Reaktor wird erst auf Drängen der Kontrollbehörden im Februar 2002 zur Inspektion vom Netz genommen.

Schweden, Juli 2006: Nach einem Kurzschluss wird im Kernkraftwerk Forsmark einer von drei Reaktoren automatisch von der Stromversorgung getrennt. Der Reaktor wird heruntergefahren.

Japan, März 2011: Im Atomkraftwerk Fukushima fallen nach einem schweren Erdbeben mit Tsunami mehrere Kühlanlagen aus, mit anschliessenden Wasserstoff-Explosionen, und es ist zu partiellen Kernschmelzen gekommen. heute noch umstritten. Nur noch wenige Gremien, darunter die WHO [8] und die IAEA [9], verharmlosen weiterhin die Folgen der Katastrophe. Alexey V. Yablokov hat sie in einem kürzlich erschienenen, sehr vollständigen Band beschrieben [10]. Nur wenn die Lügen und die Verharmlosung so weitergehen, diskutieren wir in 100 Jahren noch über die Folgen von Fukushima.

Für eine Entscheidfindung über die Strategie des vorsorglichen Gesundheitsschutzes in Sachen Atomenergie brauchen wir keine Studien mehr. Wir wissen genug, um zu handeln.

\section{Literatur}

1 Virchow R. Abhandlungen zur wissenschaftlichen Medicin. Frankfurt; 1856. Zitiert aus der 2. unv. Auflage, 1862. S. 56.

2 Gardner MJ et al. Results of case-control study of leukaemia and lymphoma among young people near Sellafield nuclear plant in West Cumbria. British Medical Journal. 1990;300:423-9.

3 Gardner Martin J. Methods and basic data of case-control study of leukaemia and lymphoma among young people near Sellafield nuclear plant in West Cumbria. British Medical Journal. 1990;300:429-34.

4 Walter M. Strahlenschutz - Argumente gegen die von der ICRP (Internationale Kommission für Strahlenschutz) vorgesehenen Lockerungen der Regeln. Schweiz Ärztezeitung. 2005;86(26):1584.

5 Parker L, Pearce MS, Dickinson HO, Aitkin M, Craft AW. Stillbirths among offspring of male radiation workers at Sellafield nuclear reprocessing plant. The Lancet. 1999; 354:1407-14.

6 Chronic Cs-137 incorporation in children's organs, Y. I. Bandazhevsky, Swiss Med Wkly. 2003;133: 488-90.

7 Chernobyl source term, atmospheric dispersion, and dose estimation, EnergyCitationsDatabase. 1 November 1989.

8 Weltgesundheitsorganisation (WHO) mit Sitz in Genf. Homepage: www.who.int/en/

9 Internationale Atomenergieorganisation mit Sitz in Wien. Homepage: www.iaea.org/

10 Yablokov AV, Nesterenko VB, Nesterenko AV. Chernobyl: Consequences of the Catastrophe for People and the Environment, Annals of the New York Academy of Sciences. 2009; Vol. 1181. 